gene. Unfortunately, in many other conditions, the underlying tissue deteriorates, or there are different cell types involved, so administrating an AAV gene therapy will not be enough to restore function. A case in point is Leber's congenital amaurosis (LCA). Although research teams at the University of Pennsylvania and University College London used the very same gene and delivery vector for treating individuals with LCA in a clinical trial, the therapy failed to achieve durable results (Nat. Biotechnol. 33, $678,2015)$.

Targeting the gene therapy in a younger population who have had less time for function to deteriorate may explain some of the greater success (median age at randomization in the treatment arm of Spark's phase 3 trial was 11 years old, compared to 16 years old in the UPenn study on LCA). But Spark also attributes its success to attention paid to preparation of the viral vector. The manufacture of voretigene neparvovec differs from that commonly used by other groups. One striking difference is the process used to purify productive AAV2 that have taken up the foreign DNA construct. Purification is important because of the limited number of receptors in the target cells at the injection site and the need to lower the number of unproductive, empty capsids, says High. "When we make our vector preps for subretinal injection, we remove all the empty capsids, which is a by-product of almost all manufacturing systems," she says. "So then, we don't have any competition with the empties in the target cells." Spark also used a construct with a unique promoter (hybrid chicken $\beta$-actin promoter with a cytomegalovirus enhancer) and an immunomodulatory regimen (the steroid prednisone) that were different from what were used in the University of Pennsylvania and University College London trials in LCA. The company even used a surfactant to prevent the capsids from adsorbing to the plastic surfaces in the delivery syringe, tubing and cannula.

The lesson for industry is that the details matter and they differ widely depending on the indication. Spark Therapeutics' High has been working on gene therapies for 25 years, and first started collaborating with Jean Bennett on RPE65 gene therapy in 2005. "She has been tinkering with the details for a quarter of a century already," says Elemer Piros, a senior biotech analyst and managing director at financial services firm Cantor Fitzgerald in New York. "So the manufacturing, the dosing, the formulation have undergone an extreme level of optimization that manifested in such clear-cut results," he says. Rather than paving a road to success, perhaps Spark Therapeutics should be seen as bushwhacking a trail, and those following in their footsteps are likely to find it still tough going. "A lot of these details were kept as trade secrets," says Piros. "So in some ways the followers would have to go through some of the same challenges that Spark did."

Spark Therapeutics' anticipated entry into the market will also force the industry to adapt pricing to one-off, potentially curative treatments. The adjustment is particularly challenging for reimbursement. There's a gap between what the reimbursement structures can handle and what the therapies are worth, says Chris Hollowood, chief investment officer for life sciences investment trust Syncona in St. Peter Port, Guernsey. There's "a disconnect between the value and the cash flow, he says.

The industry is weighing payment models, including one-time payments, an annuity model and a pay-for-performance model, says Piros. The annuity model could be problematic in the US where patients sometimes change insurance carriers, and a carrier might be reluctant to pick up the tab for a therapy approved by a previous carrier, he says. The pay-for-performance model is similar to the annuity model, but requires monitoring the effectiveness of treatments to determine if and when a payer should stop making payments. Payers are leaning toward a one-time payment for voretigene neparvovec, says Piros.

The question then becomes how much. Novartis' adoptive chimeric antigen receptor T-cell (CAR-T) therapy Kymriah, also a one-time treatment, is priced at $\$ 475,000$. But Kymriah and gene replacement therapies in the pipeline for indications, such as hemophilia, are competing as replacements for existing therapies making it possible to measure cost savings and thus the value of new treatments. Inherited retinal dystrophies, however, have no alternative treatments, so coming up with a price is trickier. Estimates for a price for voretigene neparvovec range from $\$ 500,000$ to $\$ 1.5$ million, says Piros. Given the small patient population, payers are unlikely to take a big hit from a relatively high payment price, he says. "I don't believe that there's going to be a backlash if you see a seven-figure price for this type of intervention."

The industry will need to adjust to this new class of therapies because several more are likely to emerge from the pipeline in the near future. BioMarin Pharmaceutical of San Rafael, California, is slated to begin phase 3 trials of its hemophilia therapy before the end of the year, and AveXis of Bannockburn, Illinois, has been cleared by the FDA to put their spinal muscular atrophy therapy into phase 3 trial.

\section{Duchenne drug clings on for FDA nod}

PTC Therapeutics' hopes for entering the US market for treating Duchenne muscular dystrophy (DMD) were dashed-at least in the near term-when a Food and Drug Administration Advisory Committee overwhelmingly recommended against approval of its drug candidate ataluren on September 28. Ataluren targets nonsense codons (the triplet nucleotide sequences UAA, UAG or UGA) that create premature stop signals in mRNA translation, resulting in truncated proteins. These nonsense mutations can cause a variety of genetic diseases, including DMD, beta-thalassemia and cystic fibrosis. In February 2016, the FDA refused to review PTC's application for marketing approval of ataluren; the drug had failed to meet its primary endpoint in a phase 3 trial. A subsequent meta-analysis of phase $2 \mathrm{~b}$ and phase 3 data across several endpoints, however, did show promise and gained the South Plainfield, New Jersey, biotech a conditional approval in Europe for the drug in 2016. The European regulator proposed a new three-year phase 3 study to develop further evidence regarding the drug's effectiveness. PTC also continued to petition the US regulator on the basis of the new review of its data and the promise of a new trial. Although the FDA advisors agreed that the clinical data suggested ataluren would be an effective treatment, all but one said that the post hoc nature of the evidence failed to constitute a definitive proof of efficacy. Still, patient advocates and investors in PTC are hoping that FDA will ignore the Advisory Committee's recommendation. That rarely occurs, but was the case with its review of Cambridge, Massachusetts-based Sarepta Therapeutics' DMD drug Exondys 51 (eteplirsen). After months of lobbying efforts by patients, in September 2016 Exondys gained accelerated approval in the US, against the scientific advice of FDA scientists and staff (Nat. Biotechnol. 34, 1078, 2016).

\section{C "Collectively, patient testimony today at \$ONCE [Spark Therapeutics] FDA panel ranks among most compelling I've heard, ever. Gene therapy, when it works, is... wow!" Adam Feuerstein, live tweeting from the FDA Advisory Committee panel on Spark Therapeutics' Luxturna, a one-time gene therapy meant to treat vision loss due to RPE65-mutation-associated retinal dystrophy. (STAT News, 11 October, 2017)}

\footnotetext{
"The scary part, maybe the take-home message, is that it doesn't take that muchjust one mutation-to make something really, really bad." Hongjun Song, a Zika researcher at the University of Pennsylvania, reacts to a report that a single mutation in the Zika genome may have been the reason the virus, which had been around for decades, was suddenly so harmful to fetuses. (The New York Times, 28 September 2017) 